\title{
Revisiting the assessment of tremor:
}

\author{
clinical review
}

N Vijiaratnam, FRACP, clinical research fellow, Department of Clinical and Movement Neurosciences, UCL Queen Square Institute of Neurology, University College London, London; National Hospital for Neurology and Neurosurgery, London; Department of Neurology, Royal Free Hospital, London. T Wirth, MD, MSc, clinical research fellow, Department of Clinical and Movement Neurosciences, UCL Queen Square Institute of Neurology, University College London, London; National Hospital for Neurology and Neurosurgery, London. HR Morris, PhD, FRCP, professor of neurology and consultant neurologist, Department of Clinical and Movement Neurosciences, UCL Queen Square Institute of Neurology, University College London, London; National Hospital for Neurology and Neurosurgery, London; Department of Neurology, Royal Free Hospital, London; UCL Movement Disorders Centre, UCL Queen Square Institute of Neurology, London.

\section{Address for correspondence}

Huw Morris, Department of Clinical and Movement Neurosciences, UCL Queen Square Institute of Neurology, University College London, Level 2, 33 Queen Square London, London WC1E 6BT, UK.

Email: h.morris@ucl.ac.uk

Submitted: 2 September 2020; Editor's response: 28 September 2020; final acceptance: 16 October 2020.

@British Journal of General Practice 2020; 70: 611-614.

DOI: https://doi.org/10.3399/bjgp20X713849

\section{INTRODUCTION}

Tremor, an involuntary, rhythmic, and oscillatory movement of a body part, is a frequent presenting symptom to general practice and by far the most common movement disorder presentation, impacting up to $15 \%$ of such cases. ${ }^{1}$ A common initial pattern is symmetric upper-limb involvement during posture and action. Although patients are often worried about Parkinson's disease (PD), PD tremor usually has easily recognisable features. ${ }^{2}$ This concern tends to lead to frequent referrals for specialist input despite an alternative diagnosis being more likely in a majority of cases. Essential tremor (ET) is the most common diagnosis given to patients with this presentation, which is estimated to affect $0.4-6.0 \%$ of the general population. ${ }^{3}$ This may be an overestimate as the rubric of ET and the relationship between clinical features and underlying pathophysiology is uncertain. These aspects also potentially contribute to variable diagnostic and treatment outcomes. ${ }^{4} \mathrm{~A}$ recent Movement Disorder Society consensus on phenotyping charts a course towards more precise classification. ${ }^{5}$ This will not only be useful for research but also help in clarifying common clinical syndromes seen in everyday practice. This article outlines an approach to upperlimb tremor presentations in adult patients, developing a previously proposed three cardinal question method for neck pain while highlighting salient aspects of the consensus statement that could potentially aid in clinical stratification of cases.

\section{CLINICAL ASSESSMENT \\ History}

Characterising the onset age, anatomical distribution (head, voice, hands, and symmetryl, and temporal evolution of the tremor is important, forms the first step of the assessment, and aids in determining the underlying syndrome loutlined in subsequent sections). A key aspect to establish early is if the tremor results in functional impairment as this will determine if treatment is warranted lall current treatment options are symptomatic, that is, non-disease modifying). ${ }^{7.8}$ Useful questions to establish this include when the tremor is worst (writing, using a cup, relaxing) and whether assistance is needed, for example, with preparing food. Then, relevant family and past histories (medication exposure, alcohol, and drug sensitivity) as well as the presence of other neurological symptoms (for example, slowing up, gait disturbance, muscle stiffness, and aches) should be explored. The latter aspects are more typical of PD than ET and can provide early diagnostic clues in differentiating these two conditions. Some tremorgenic medications to consider include salbutamol, ciclosporin, haloperidol, lithium, and valproic acid. Caffeine intake, anxiety, and alcohol use can result in or exacerbate pre-existing tremor and these aspects should be clarified on history taking. However, some forms of tremor are alcohol responsive so alcohol misuse may be secondary to an underlying neurological condition.

\section{Examination}

Observation is key and should continue throughout the consultation. This includes the patient's gait and movements when walking into the room, particularly noting how easily they stand up (difficulty in parkinsonian syndromes), how far apart their feet are (broad stance implicates potential proprioceptive or cerebellar impairment), and if they take appropriately spaced strides (short strides in parkinsonian syndromes). Other parkinsonian features lreduced blinking, decreased facial expression, stooped posture), the location of the tremor, and the presence of systemic signs (exophthalmos, goitre) may also be evident before the formal examination.

Defining tremor characteristics (frequency, amplitude, and nature) is of little value though establishing changes in activation conditions is useful. This includes assessments at 
A

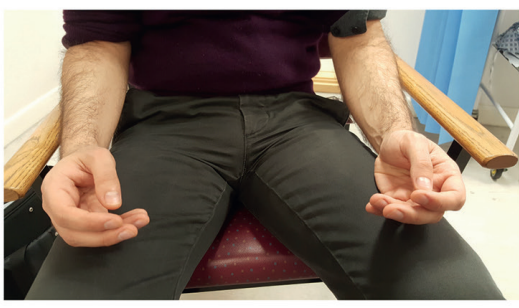

B

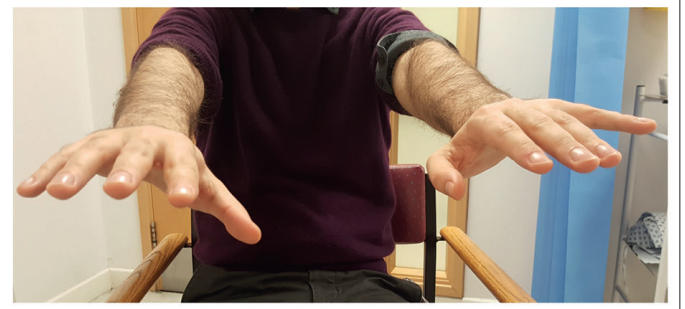

C

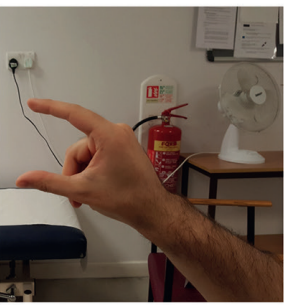

D

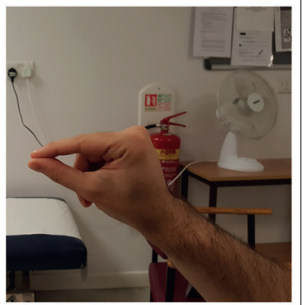

$\mathbf{E}$

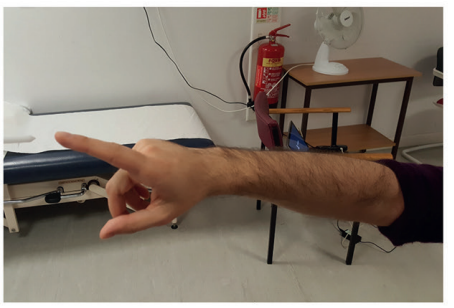

rest - limbs supported on the patient's lap (Figure 1A) and observing for tremor with distraction lcounting backwards or complex subtractions), which is typical of PD tremor. Postural tremor is then assessed with both upper limbs outstretched (Figure 1B) and followed by the finger-nose task (Figure $1 E)$, looking for a kinetic or intention tremor. During the transition from rest to posture, re-emergent tremor (that is, the tremor disappears and then reappears) should be established (strongly suggestive of PD). Additional movements (dystonia or myoclonus) should also be noted. Definitions are outlined in Box 1.

Coexistent limb parkinsonism can be detected with finger taps (Figure 1C, D). Patients tap their thumb and index finger approximately 10 times. Small and slow movements suggest bradykinesia whereas taps progressively becoming smaller (decrement) or stopping imply PD. Rotation of the hand at the wrist to note for resistance lextrapyramidal tone) follows. Handwriting to

Figure 1. Physical examination of tremor. ${ }^{a}$ ${ }^{a}$ The tremor is first assessed at rest, palm up with upper-limb support on the patient's lap (A). A postural tremor is then searched for by asking the patient to outstretch their arms (B). A bradykinesia, suggestive of an extrapyramidal syndrome, can be identified by asking the patient to tap the finger IC and D). Eventually, an intention tremor is better shown by the nose-finger test (E). assess for micrographia (small handwriting progressively gets smaller) or writing tremor and functional impairment (for example, pouring water from cup to cup) completes the examination. Exacerbation (or provocation) of tremor when pouring water from cup to cup is typical of essential and dystonic tremor.

\section{Investigations}

Tests to exclude symptomatic causes (thyroid, renal, and liver function) should be performed in primary care. Additional tests may be pertinent but should be considered by specialists.

\section{SYNDROMES, AETIOLOGIES, AND RED FLAGS}

A recent expert consensus proposal suggests an axes approach to organising clinical findings. ${ }^{5}$ This could potentially aid in conceptualising cases in practice rather than focusing purely on a diagnostic label, though detailed utility of this approach would more likely be relevant in neurological practice. Axis 1 comprises efforts to define clinical features (that is, a syndrome observed based on the history and examination) and axis 2 encompasses testing to determine the underlying aetiology. Axis 1 findings can reflect characteristic syndromes isolated tremor syndrome (when monosymptomatic) or combined tremor syndrome (when the tremor is associated with other clinical signs). The axis 2 cause identified is the underlying diagnosis rather than the syndrome defined. Both axis features should be regularly re-evaluated if initially not characteristic of an established diagnostic entity. Different axis 2 aetiologies resulting in one or a variety of axis 1 syndromes are possible, and the converse holds true.

This article next considers upper-limb syndromes, highlighting recent definition changes and features for referral.

\section{Essential tremor}

Essential tremor is an isolated bilateral upper-limb action tremor syndrome. Although coexistent tremor (head, voice, lower limbs) can occur, isolated tremor in these regions should not be regarded as ET. ${ }^{5}$ Useful diagnostic features include tremor during action, including writing, holding a cup and saucer and pouring, together with the absence of bradykinesia. A 3-year observation period during which the tremor is considered indeterminate has been proposed. This is to safeguard against the development of additional features and therefore alternative syndromes. If the onset is sudden and deterioration stepwise this should prompt clinicians away from ET. Although responses to alcohol, propranolol, and a family history are sometimes evident, these are not reliable diagnostic features. ${ }^{5}$ The presence of other neurological signs, even if subtle or uncertain, constitute the new entity essential tremor plus (ET-plus) ${ }^{5}$ and warrant neurological input.

\section{Essential tremor plus}

Patients with ET-plus have ET features with additional signs that are subtle and their presence at times questionable. Dystonia is the most common of these and may include minimal flexion posturing of the fingers/wrist or pronation/supination tilting of the outstretched hands. This group either represent novel disorders or alternative conditions (dystonic tremor, PD) in their infancy. Although diagnostic value is currently lacking, practitioners are encouraged to attempt characterisations and consider referral as it could potentially alter future outcomes. 


\section{Box 1. Summary of terminology and definitions}

\begin{tabular}{|c|c|}
\hline $\begin{array}{l}\text { Tremor } \\
\text { terminology }\end{array}$ & Definition \\
\hline \multicolumn{2}{|l|}{$\begin{array}{l}\text { Anatomical } \\
\text { distribution }\end{array}$} \\
\hline Focal & One body region affected \\
\hline Segmental & $\begin{array}{l}\text { Two or more } \\
\text { contiguous body parts } \\
\text { affected }\end{array}$ \\
\hline Hemi & $\begin{array}{l}\text { One side of body } \\
\text { affected }\end{array}$ \\
\hline Generalised & $\begin{array}{l}\text { Upper and lower body } \\
\text { affected }\end{array}$ \\
\hline Orthostatic & $\begin{array}{l}\text { Lower limbs or } \\
\text { trunk affected during } \\
\text { standing }\end{array}$ \\
\hline \multicolumn{2}{|l|}{$\begin{array}{l}\text { Activation } \\
\text { conditions }\end{array}$} \\
\hline Rest & $\begin{array}{l}\text { Tremor in a body part } \\
\text { that is not voluntarily } \\
\text { activated }\end{array}$ \\
\hline Action & $\begin{array}{l}\text { Tremor occurring } \\
\text { while voluntarily } \\
\text { maintaining a position } \\
\text { against gravity (can be } \\
\text { re-emergent, postural, } \\
\text { or orthostatic) or during } \\
\text { movement (kinetic) }\end{array}$ \\
\hline $\begin{array}{l}\text { Position-specific } \\
\text { postural }\end{array}$ & $\begin{array}{l}\text { Tremor when } \\
\text { maintaining a specific } \\
\text { position or posture }\end{array}$ \\
\hline Kinetic (simple) & $\begin{array}{l}\text { Tremor roughly the } \\
\text { same throughout } \\
\text { movement }\end{array}$ \\
\hline Kinetic (intention) & $\begin{array}{l}\text { Crescendo increase } \\
\text { of tremor on nearing } \\
\text { target }\end{array}$ \\
\hline $\begin{array}{l}\text { Kinetic (task } \\
\text { specific) }\end{array}$ & $\begin{array}{l}\text { Tremor occurring } \\
\text { during a specific task } \\
\text { (for example, writing) }\end{array}$ \\
\hline \multicolumn{2}{|l|}{ Other movements } \\
\hline Dystonia & $\begin{array}{l}\text { Involuntary twisting and } \\
\text { abnormal posturing of } \\
\text { a muscle or group of } \\
\text { muscles }\end{array}$ \\
\hline Myoclonus & $\begin{array}{l}\text { Brief, involuntary, } \\
\text { irregular (lacking } \\
\text { rhythm) twitching of a } \\
\text { muscle or a group of } \\
\text { muscles }\end{array}$ \\
\hline
\end{tabular}

\section{Enhanced physiological tremor}

Enhanced physiological tremor (EPT) resembles ET and occurs because of an enhancement of normal physiology by precipitating conditions lanxiety, drugs, and thyroid disease). ${ }^{9}$ EPT tends to vary with these factors and is not functionally limiting The proposed 3-year ET observation period potentially aids in delineation and reversal of causes.

\section{Tremor combined with parkinsonism}

Classical parkinsonian tremor manifests with a 4-7 Hz asymmetrical hand tremor, resting 'pill-rolling' tremor with associated bradykinesia (with decrement), and rigidity. Lower-limb, jaw, tongue, or foot tremors can variably coexist. $^{5}$ Postural/kinetic (action) tremor resembling ET can be a presenting feature of PD. These patients should therefore be regularly re-examined for emergence of Parkinsonism. PD tremor usually abates with action or assuming a position, with re-emergence with sustained posture (re-emergent tremor). ${ }^{10}$

\section{Isolated focal tremors}

Focal tremors affect the head, voice, palate, or limbs and occur in isolated conditions or during specific tasks such as writing or while playing instruments or sport. ${ }^{11-13}$ These tremors potentially reflect underlying dystonia and should not be regarded as ET. 5,14 They can at times be attributed to an underlying lesion ${ }^{14,15}$ or suggest a genetic abnormality. ${ }^{16}$

Isolated rest tremor without parkinsonism should either prompt careful clinical surveillance or imaging for dopaminergic deficiency. ${ }^{17}$ If rest tremor occurs in conjunction with an ET phenotype (typically in advanced stages) and in the absence of parkinsonism, this constitutes ET-plus. ${ }^{5}$ Neurophysiological assessments can aid in distinguishing PD from ET rest tremor.18 Orthostatic tremor (OT) is an isolated tremor occurring mainly in patients' lower limbs when standing. Although coexistent upperlimb or head tremors can occur, ${ }^{19,20}$ this should not be regarded as ET. Diagnostic delays have been noted ${ }^{19}$ as initial symptoms can be non-specific (legs feeling like jelly, unsteadiness). These features should therefore prompt a clinical suspicion for OT.

\section{Dystonic tremor syndromes}

If a tremor occurs in a body part affected by dystonia this is termed dystonic tremor (DT) Tremor and dystonia occurring in different body regions in contrast are defined as tremor associated with dystonia., ${ }^{5,21}$ DTs are typically variable, and are jerky. ${ }^{21}$ Other distinguishing signs exist ${ }^{21}$ though detection would not be expected in general practice. The distinction between DT and ET-plus with dystonia currently solely relies on the confidence of a clinician in apportioning dystonia though neurophysiology may change this in the future. Patients with DT can present with an isolated head tremor and/or associated head and neck posturing (cervical dystonia). This can occur in isolation or in combination with an asymmetrical upper-limb tremor. DT's variable nature can sometimes lead to a misdiagnosis of a functional tremor. Specialist input should therefore be sought in these cases nor only to clarify first the diagnosis but also for consideration of specific efficacious treatments such as botulinum toxin therapy or functional neurosurgery.

\section{Intention tremor syndromes}

Previously classified as cerebellar tremor, these cases are now considered as either isolated or combined Itypically dystonia or cerebellar signs) intention tremor syndromes. Intention tremors are typically $<5 \mathrm{~Hz}$ with an underlying cause in the brainstem-cerebello-thalamic pathway. ${ }^{5}$

\section{Functional tremor}

Functional tremor is diagnosed with the demonstration of 'positive' features (sudden onset and inconsistencies, fluctuations, distractibility, and entrainability on examination). Although a coexisting psychiatric history in particular anxiety can be present, it should not be relied upon. ${ }^{22}$ Neurophysiology can sometimes aid the diagnosis, ${ }^{23}$ which should be established by a specialist.

\section{THREE 'CARDINAL' QUESTIONS CLINICAL APPROACH TO TREMOR}

A previously proposed approach of three 'cardinal' questions to address neck pain could potentially be useful when considering tremor. ${ }^{6}$ The first consideration is if the complaint is an emergency, though these presentations of tremor are rare. An abrupt onset and rapid progression (days to weeks) are concerning. However, rapidly progressive tremor with other neurological features such as dysarthria, ataxia, seizures, or a progressive gait and balance disorder warrants urgent neurological evaluation. ${ }^{24}$ This may relate to an acute toxic (druginduced) process or an immune-mediated process. Immune-mediated disorders can occur because of an underlying neoplasm, and result in significant long-term sequelae, although early diagnostics and treatment can substantially alter outcomes. ${ }^{24}$ Toxic medication levels (for example, lithium, valproic acid) are an alternative possibility. 


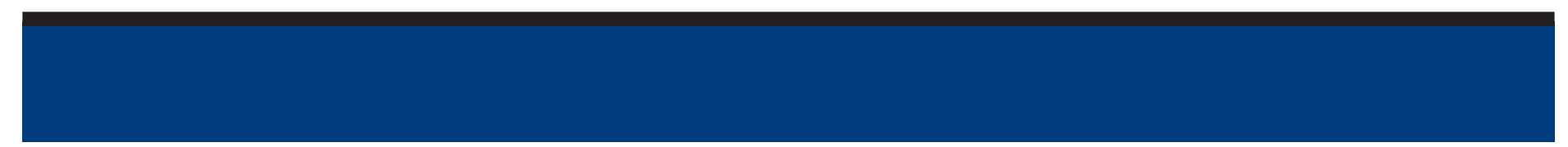

A thorough medication history, serum drug levels, and an emergency admission should therefore be considered in cases with these features.

The next question is if further investigations or treatments are required in primary care. It is proposed that patients with isolated upperlimb action tremor should have blood tests to exclude reversible causes (renal function, thyroid function). In young-onset cases $(<40)$ (all syndromes), Wilson's disease should be excluded with biochemical testing (liver function tests, serum ceruloplasmin, and copper). In the absence of reversible causes and the presence of functional impairment. practitioners could consider initiating propranolol or primidone. In convincing PD cases with functional impairment trialling levodopa may be worthwhile while awaiting specialist input, depending on local referral patterns and practice.

The final consideration is if the patient requires specialist input. It is proposed that

\section{REFERENCES}

1. Wenning GK, Kiechl S, Seppi K, et al. Prevalence of movement disorders in men and women aged 50-89 years (Bruneck Study cohort): a population-based study. Lancet Neurol 2005; 4(12): 815-820.

\section{Funding}

None.

\section{Provenance}

Freely submitted; externally peer reviewed.

\section{Competing interests}

Nirosen Vijiaratnam has received unrestricted educational support from AbbVie, Stada, Ipsen, and Merck, and speaker's honorarium from AbbVie and Stada. Dr Vijiaratnam's current research position is supported by the Janet Owen's charitable foundation. Huw R Morris is employed by UCL. In the last 24 months he reports paid consultancy from Biogen, UCB, AbbVie, Denali, Biohaven, Lundbeck; lecture fees/honoraria from Biogen, UCB, C4X Discovery, GE Healthcare, Wellcome Trust, Movement Disorder Society; research grants from Parkinson's UK, Cure Parkinson's Trust, PSP Association, CBD Solutions, Drake Foundation, Medical Research Council. Dr Morris is a co-applicant on a patent application related to C90RF72 - Method for diagnosing a neurodegenerative disease (PCT/GB2012/052140). Thomas Wirth has received grants from the association APTES' and the 'Fondation Planiol' Study

\section{Discuss this article}

Contribute and read comments about this article: bjgp.org/letters all cases with a clinical syndrome rather than ET should be referred. This is to establish the diagnosis with certainty while facilitating specialised treatments (for example, botulinum toxin and functional neurosurgeryl. ET cases refractory to medication with functional limitation would also benefit from specialist input for diagnostic validation and alternative treatment consideration. Functional neurosurgery for severe, refractory upper-limb tremor and botulinum toxin treatment for head tremor may be very effective in selected cases.

\section{CONCLUSION}

This article outlines a clinical approach to upper limb tremor. The emphasis is to regard tremor as a symptom and to focus on defining a syndrome rather than presuming a diagnosis. This concept is particularly crucial when considering the common clinical syndrome of ET.

12. Lee A, Furuya S, Altenmüller E. Epidemiology and treatment of 23 musicians with task specific tremor. J Clin Mov Disord 2014; 1: 5.

13. Dhungana S, Jankovic J. Yips and other movement disorders in golfers. Mov Disord 2013; 28(5): 576-581.

14. Rivest J, Marsden CD. Trunk and head tremor as isolated manifestations of dystonia. Mov Disord 1990; 5(1): 60-65.

15. Zadikoff C, Lang AE, Klein C. The 'essentials' of essential palatal tremor: a reappraisal of the nosology. Brain 2006; 129(Pt 4): 832-840.

16. Stamelou M, Charlesworth G, Cordivari C, et al. The phenotypic spectrum of DYT24 due to ANO3 mutations. Mov Disord 2014; 29(7): 928-934.

17. Scherfler C, Schwarz J, Antonini A, et al. Role of DAT-SPECT in the diagnostic work up of parkinsonism. Mov Disord 2007; 22(9): 1229-1238.

18. Nisticò R, Pirritano D, Salsone M, et al. Synchronous pattern distinguishes resting tremor associated with essential tremor from rest tremor of Parkinson's disease. Parkinsonism Relat Disord 2011; 17(1): 30-33.

19. Hassan A, Ahlskog JE, Matsumoto JY, et al. Orthostatic tremor: clinical, electrophysiologic, and treatment findings in 184 patients. Neurology 2016; 87(3): 341.

20. Vijiaratnam N, Sirisena D, Paul E, et al. Measuring disease progression and disability in orthostatic tremor. Parkinsonism Relat Disord 2018; 55: 138-140.

21. Elble RJ. Defining dystonic tremor. Curr Neuropharmacol 2013; 11(1): 48-52.

22. Hallett M. Physiology of psychogenic movement disorders. J Clin Neurosci2010; 17(8): 959-965.

23. Schwingenschuh $P$, Saifee TA, KatschnigWinter P, et al. Validation of 'laboratorysupported' criteria for functional (psychogenic) tremor. Mov Disord 2016; 31(4): 555-562.

24. Baizabal-Carvallo JF, Jankovic J. Autoimmune and paraneoplastic movement disorders: an update. J Neurol Sci 2018; 385: 175-184. 